\title{
First Principles Study on Electronic Structure and Optical Properties of PMMA Doped InSb-Mn Alloy Polymer Matrix Composite
}

\author{
Dhanabalakrishnan Kovilpalayam Palaniswamy, ${ }^{1}$ Pandiyan Arumugan, ${ }^{2}$ \\ Ravindiran Munusami, ${ }^{3}$ A Chinnasamy, ${ }^{4}$ S. Madhu, ${ }^{5}$ \\ Prathap Paulraj, ${ }^{6}$ and Kumaran Palani (iD ${ }^{7}$ \\ ${ }^{1}$ Department of Aeronautical Engineering, Hindustan Institute of Technology, Coimbatore 641032, India \\ ${ }^{2}$ Department of Mechanical Engineering,Saveetha School of Engineering, Saveetha Institute of Medical and Technical Sciences, \\ Chennai 602105, India \\ ${ }^{3}$ Department of Medical Electronics,Saveetha Engineering College, Anna University, Chennai 602105, India \\ ${ }^{4}$ Department of Computer Science and Engineering,Sri Sairam Engineering College, Anna University, Chennai, India \\ ${ }^{5}$ Department of Automobile Engineering,Saveetha School of Engineering, Saveetha Institute of Medical and Technical Sciences, \\ Chennai 602105, India \\ ${ }^{6}$ Department of Mechanical Engineering, Sri Krishna College of Technology, Coimbatore 641042, India \\ ${ }^{7}$ Department of Mechanical Engineering, College of Engineering, Wolaita Sodo University, Wolaita Sodo, Ethiopia
}

Correspondence should be addressed to Kumaran Palani; pkumaran2003et@gmail.com

Received 10 October 2021; Revised 13 November 2021; Accepted 23 December 2021; Published 11 January 2022

Academic Editor: V. Vijayan

Copyright (c) 2022 Dhanabalakrishnan Kovilpalayam Palaniswamy et al. This is an open access article distributed under the Creative Commons Attribution License, which permits unrestricted use, distribution, and reproduction in any medium, provided the original work is properly cited.

\begin{abstract}
InSb the group III-V semiconductor with narrow band gap is combined with $\mathrm{Mn}$ in various concentrations and that InSb-Mn alloy is doped with poly methyl methacrylate (PMMA). The optical properties and electronic structure of ternary InSb-Mn alloy with PMMA are investigated by first principles calculations using the DFT method. Varying Mn concentrations play an important role in the improvement of the absorption coefficient and optical conductivity. It is observed that the band gap of InSb-Mn: PMMA decreases monotonously with the increase in Mn concentration. Optical properties of InSb-Mn: PMMA, such as the optical absorption coefficient and optical conductivity, are greater than those of pure InSb. InSb-Mn: PMMA alloy is doped with PMMA polymer in order to make a thin film as PMMA is a transparent thermoplastic polymer. These results suggest a promising application of InSb-Mn: PMMA thin film in optoelectronics when the InSb doping is $24 \%$ with improved conductivity when compared with other doping ratios. This states the optimum doping ratio and the major finding in the carried out research based on modelling and simulation.
\end{abstract}

\section{Introduction}

III-V semiconductors, because of their structure, play a crucial role in scientific research and its application. Preparation of the device of compound semiconductors particularly of III-V group elements has improved in recent years as these compounds are proven technologically that these are highly helpful for galvanometric devices, high-speed electronics, magnetic sensors, and infrared detectors in distinct wavelength ranges [1-3]. Some of group III-V compounds such as GaAs, InP, InSb, GaSb, and AlSb have high electron mobility that makes them helpful for many photonic applications. InSb and doped InSb semiconductor are widely implemented in infrared detectors and highspeed devices because of narrow band gap [4] of $0.17 \mathrm{eV}$ at $300 \mathrm{k}$ and $0.23 \mathrm{eV}$ at $80 \mathrm{k}$ and additionally its high electron 
mobility of $80,000 \mathrm{~cm}^{2} / \mathrm{Vs}$. A p-type InSb is employed in magnetic sensitive devices like magneto resistors [5]. Applications could include ultrahigh-speed and ultralow power integrated circuits, satellite communications, active-array space-based radar, portable mobile devices, chemical detection, drug analysis, gas environmental monitoring, biomedical diagnosis, and other fields $[6,7]$. The leading advancement in thin film materials and devices has initialized many of developments in field of flexible electronics including polymers, nanowires, carbon nanotubes, or other nanomaterials with semiconducting thin films enabling the enhancement in their properties $[8,9]$ Devices based on such composites show improvements in electrical performance. In recent years, there is holding attention in the characterization of InSb thin film because of its potential optoelectronics applications in various fields. The extreme range of application of InSb is required in the form of thin film. InSb films have associate important application in device manufacturing, applied in position detectors and drive motors part of equipment [10-14]. In this paper, we propose a polymer thin film made of InSb-Mn and poly methyl methacrylate (PMMA) for improved conductivity and absorption. PMMA is a thermoplastic material with outstanding optical and mechanical properties supporting wide spread applications such as photovoltaics, laser power attenuators, blue light filters, biomedical (bone cement), and optical data storage [15-21]. Optical property of the InSb-Mn with the PMMA doping improves with varying doping ratios [22]. Experimental validation and the process involved make it time consuming to check any composite. Hence, the gap in the process was addressed in the present work to cut short the time and to identify the optimum doping concentration before even going for experiment.

\section{Methodology}

Methodology of the proposed work involves the first principle calculation by density functional theory (DFT). Cambridge serial total energy package (CASTEP) was used to calculate the DFT using the plane wave pseudo potential method for calculating the properties of materials from the first principles. Calculations were performed to simulate various properties of materials such as energetics, electronic response properties, vibrational properties, and structure at the atomic level $[23,24]$. The concept of a pseudo potential is an important one for plane wave total energy methods since the alternative full Coulomb potential of the electron-ion interaction decays too slowly to be accurately represented by a small number of Fourier components. The most general form for a pseudo potential is represented in the following equation [25]:

$$
\mathrm{NL}=\sum\left|\operatorname{lm}>\mathrm{V}_{l}<\operatorname{lm}\right| \text {. }
$$

Generalized gradient approximation (GGA) with Perdew, Burke, and Ernzerhof (PBE) [26] parameterization was used to describe the exchange-correlation interaction. Ultrasoft pseudo-potentials [27] were applied to model the electron-ion interaction. In this paper, we mainly focus on the electronic structure and the optical properties of
InSb-Mn. The DFT algorithm was chosen for the geometry optimization of the super-cells. The energy cut-off for the plane wave basis was chosen as $300 \mathrm{eV}$ for the electronic structure calculation and optical properties calculation. The SCF tolerances were set as follows: $2.0 \mathrm{e}^{-6} \mathrm{eV} /$ atom for the total energy; maximum SCF cycles were set as 100 . K-points were separated by $0.051 / \AA$. Augmentation density scaling factor is 1.0. Perdew, Burke, and Ernzerhof (PBE) presented a simplified construction of a simplified GGA for exchange and correlation, in which all parameters other than those in LSD are fundamental constants.

$$
F_{x}^{\mathrm{PBE}}(s)=1+k-k /\left(1+\mu s^{2} / k\right),
$$

where $\mu=0.21951$ and $k=0.804$.

\section{Calculation of Properties Using DFT}

3.1. Optical Conductivity. Another mostly used quantity for expressing optical properties is optical conductivity $\sigma(\omega)$ given as follows:

$$
\sigma=\sigma_{1}+i \sigma_{2}=-i \omega / 4 \pi(\varepsilon-1) .
$$

Optical conduction is sometimes used to characterize metals, but CASTEP is aimed toward the optical properties of insulators and semiconductors. Difference between the insulators and semiconductors is the intraband transitions which play vital role within the IR a part of the optical spectra of metals and these transitions are not considered in CASTEP [28].

3.2. Optical Absorption. In vacuum, $\mathrm{N}$ (refractive index) is real with the value being unity. For transparent materials, the real part is presented, and the imaginary part is represented as

$$
\eta=\frac{2 k \omega}{c}
$$

The coefficient of absorption indicates the fraction of energy lost by the wave once it passes through material [28]. The light absorption coefficient expresses the percentage of light intensity attenuation per units of distance traveling in the medium. Figure 1 shows that InSb with different $\mathrm{Mn}$ content all have peaks between $2.5 \mathrm{eV}$ and $5.5 \mathrm{eV}$.

3.3. Density of States. The CASTEP Density of States Options dialog allows you to specify the k-point set and the eigenvalue convergence criterion used for density of states calculations. Gamma point only, when selected, indicates that a single k-point at $(0,0,0)$ will be used for the density of states calculation [29].

3.4. Band Structure. CASTEP calculations using both the GGA and PBE [29] were performed. Pseudo-potentialmixed plane wave Gaussian basis LDA and GGA [30] and full-potential nonorthogonal local-orbital minimum basis band-structure method within the LDA were used for the calculation of the band structure [31]. 

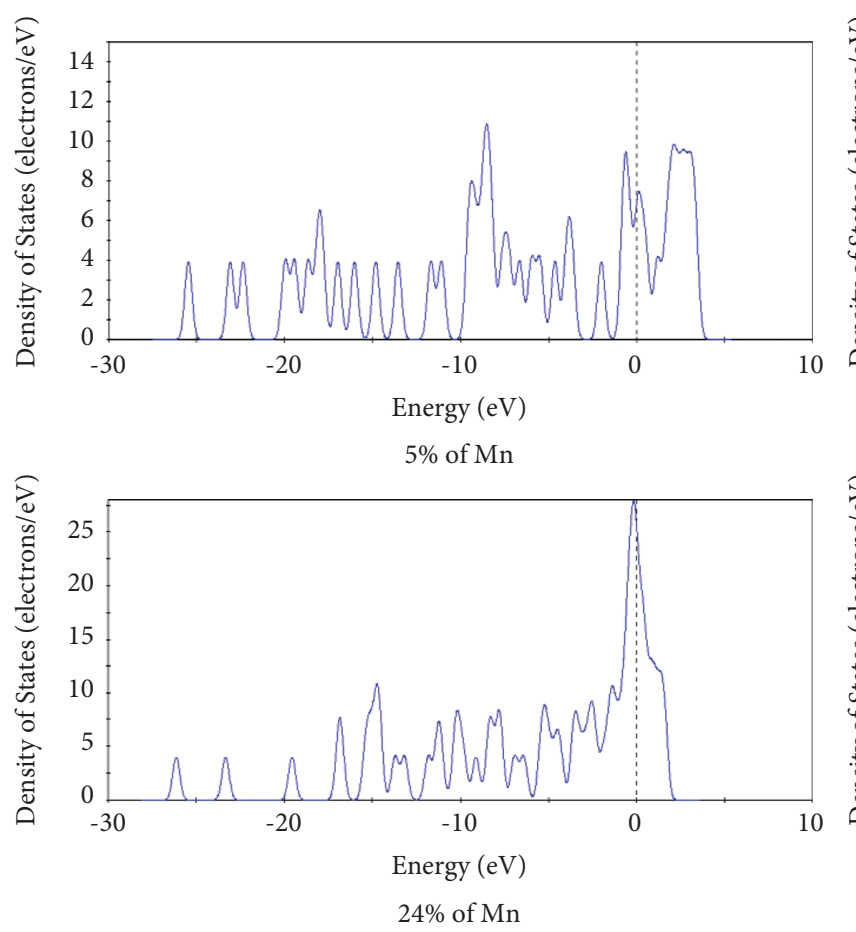
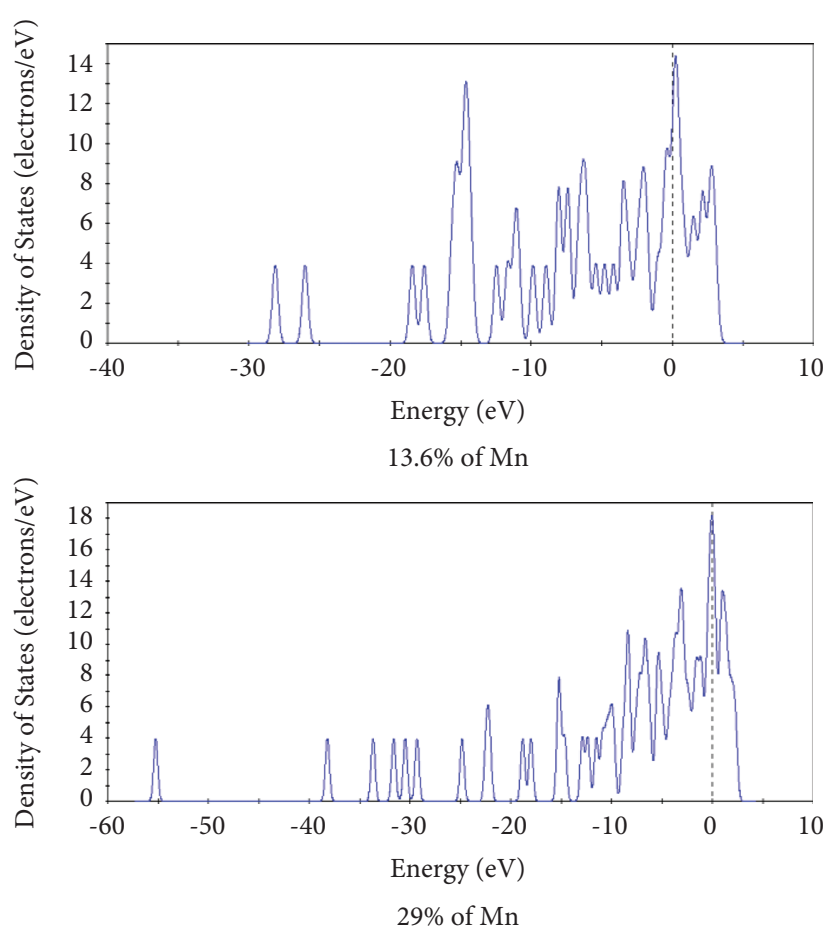

Figure 1: The density of states of InSb-Mn with $\mathrm{Mn}=5 \%, \mathrm{Mn}=13.6 \%, \mathrm{Mn}=24 \%$, and $\mathrm{Mn}=29 \%$.

\section{Results and Discussion}

Results obtained by the above CASTEP-based calculation are represented in the graphs as shown in Figures 1-4. The plot of the graphs obtained was by the material studio software package using the CASTEP-based calculations. Illustration of the graphs from the CASTEP calculations is very useful to identify the maximum optimum values of each graphically. Band structure shows the occupancy of the electrons in the sub bands and the orbitals. Optical conductivity shows the peak intensity of the composite with the doping ratios, and the absorption graph shows the maximum intensity of the absorption of the composite and its bandwidth .

4.1. Band Width. Figure 2 shows the band structure of InSb-Mn: PMMA with different Mn concentrations, it is evident that InSb is a narrow band gap semiconductor, and the band gap shows a decreasing trend as the Mn concentration increases. The result is given in Table 1.

The coordinates of the special k-points in Figure 2 are $G$ (0.000, 0.000, 0.000), F (0.000, 0.500, 0.000), $Q(0.000,0.500$, $0.500)$, and $Z(0.000,0.000,0.500)$.

InSb-Mn doped with PMMA with $24 \% \mathrm{Mn}$ has the least band gap and then $5 \%$ of $\mathrm{Mn}, 13.6 \%$ of $\mathrm{Mn}$, and $29 \%$ of $\mathrm{Mn}$. As the concentration of $\mathrm{Mn}$ is increased from $5 \%$ to $24 \%$, the band gap is decreased from then, while as the concentration of $\mathrm{Mn}$ increases, the band gap also increases. Narrowing of band gap on doping is a normal phenomenon in semiconductors. Donor impurities produce energy levels within the band gap close to the conduction band, and acceptor impurities produce energy levels close to the valence band. With the increase in the quantity of doping, the density of states of those dopants increases and forms a continuous state similar to the bands and effectively the band gap decreases. It is possible to extend the band gap of narrow band gap semiconductor by alloying it with a wide band gap semiconductor.

4.2. Optical Properties. The optical properties of InSb-Mn: PMMA mainly refer to the absorption coefficient and optical conductivity, which are primarily decided by the bands near the Fermi energy level, the concentration, and the mobility of carrier.

4.3. Absorption. Figure 3 represents the absorption of the composite with the varying doping ratio of InSb into the PMMA. InSb-Mn: PMMA with 24\% Mn has increased absorption when compared to the $5 \%$ and $13.6 \%$, whereas $29 \% \mathrm{Mn}$ concentration has a much reduced absorption. Recorded first peak is at $5 \mathrm{eV}$ and continues till $7.5 \mathrm{eV}$, and there is a further increase in at $12 \mathrm{eV}$ for the $\mathrm{Mn}$ concentration of $13.6 \%$. Recorded maximum absorption is at $24 \% \mathrm{Mn}$ concentration, whereas the Mn concentration at $29 \%$ has resulted in the peak at $4 \mathrm{eV}$ and decreases till 20 $\mathrm{eV}$.

4.4. Conductivity. Figure 4 represents the optical conductivity of the prepared composite with the varying doping concentration. The change in the color illustration shows the real (blue) and imaginary (red) parts of the recorded data in the graphical representation. Semiconductor optical 

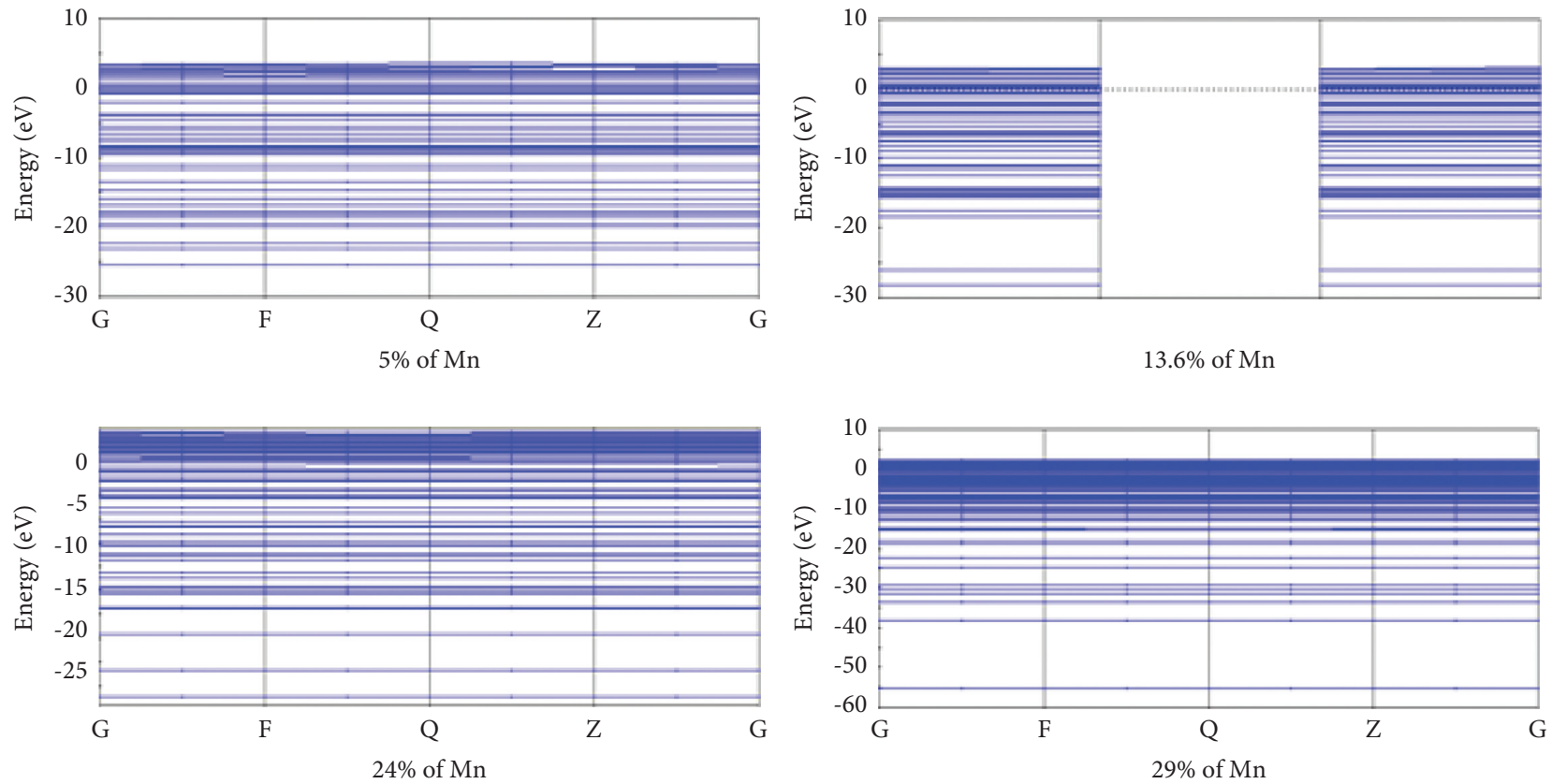

Figure 2: The band structure of InSb-Mn with $\mathrm{Mn}=5 \%, \mathrm{Mn}=13.6 \%, \mathrm{Mn}=24 \%$, and $\mathrm{Mn}=29 \%$.
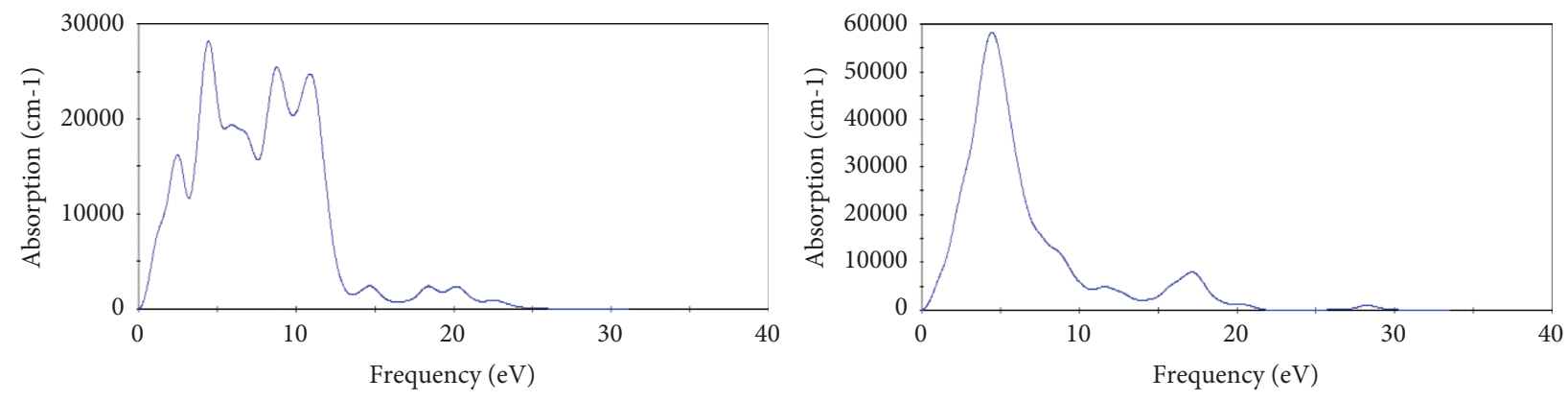

$5 \%$ of $\mathrm{Mn}$
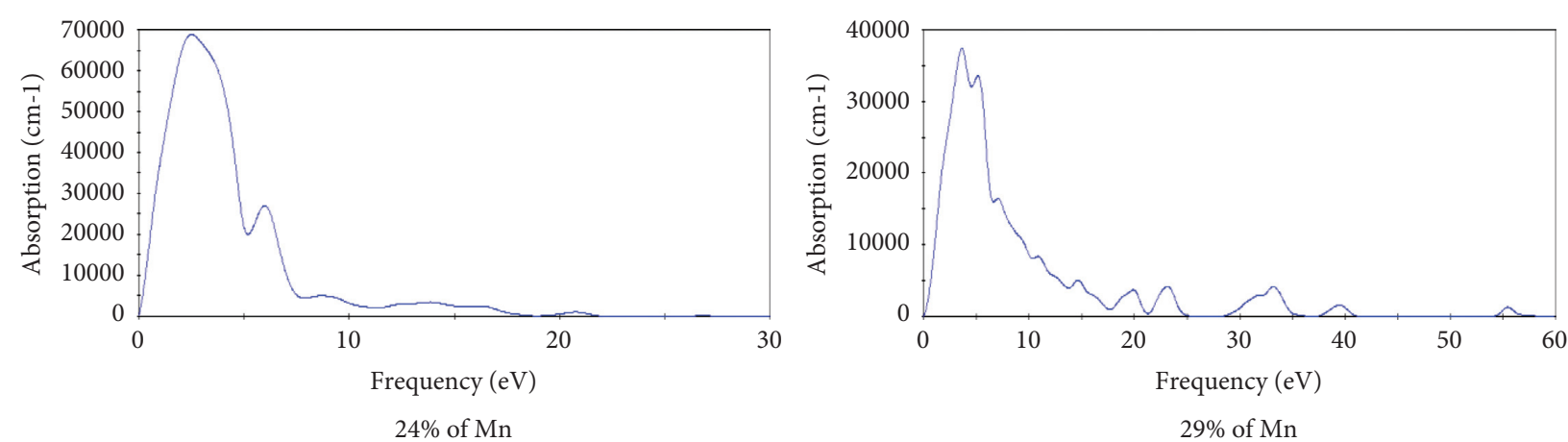

Figure 3: The absorption of InSb-Mn with $\mathrm{Mn}=5 \%, \mathrm{Mn}=13.6 \%, \mathrm{Mn}=24 \%$, and $\mathrm{Mn}=29 \%$.

conductivity is the change in conductivity caused by illumination, either an increase or a decrease. Generation and recombination effects in the material occur due to the photon effect on the materials which causes the covalent bonds to break and create new electron hole pairs. This effect makes more carriers to migrate from one place to another which causes the conductivity. The photoconductive effect is the physical basis of optoelectronic applications of semiconductors, InSb-Mn: PMMA with $24 \% \mathrm{Mn}$ has the much increase in the conductivity than $5 \%$ of $\mathrm{Mn}$ and $13.6 \%$ of $\mathrm{Mn}$ concentrations but further increase in the concentration of Mn to $29 \%$, and then there is fall in the conductivity. It is showing maximum conductivity at $24 \%$ of Mn concentration in thin film. 

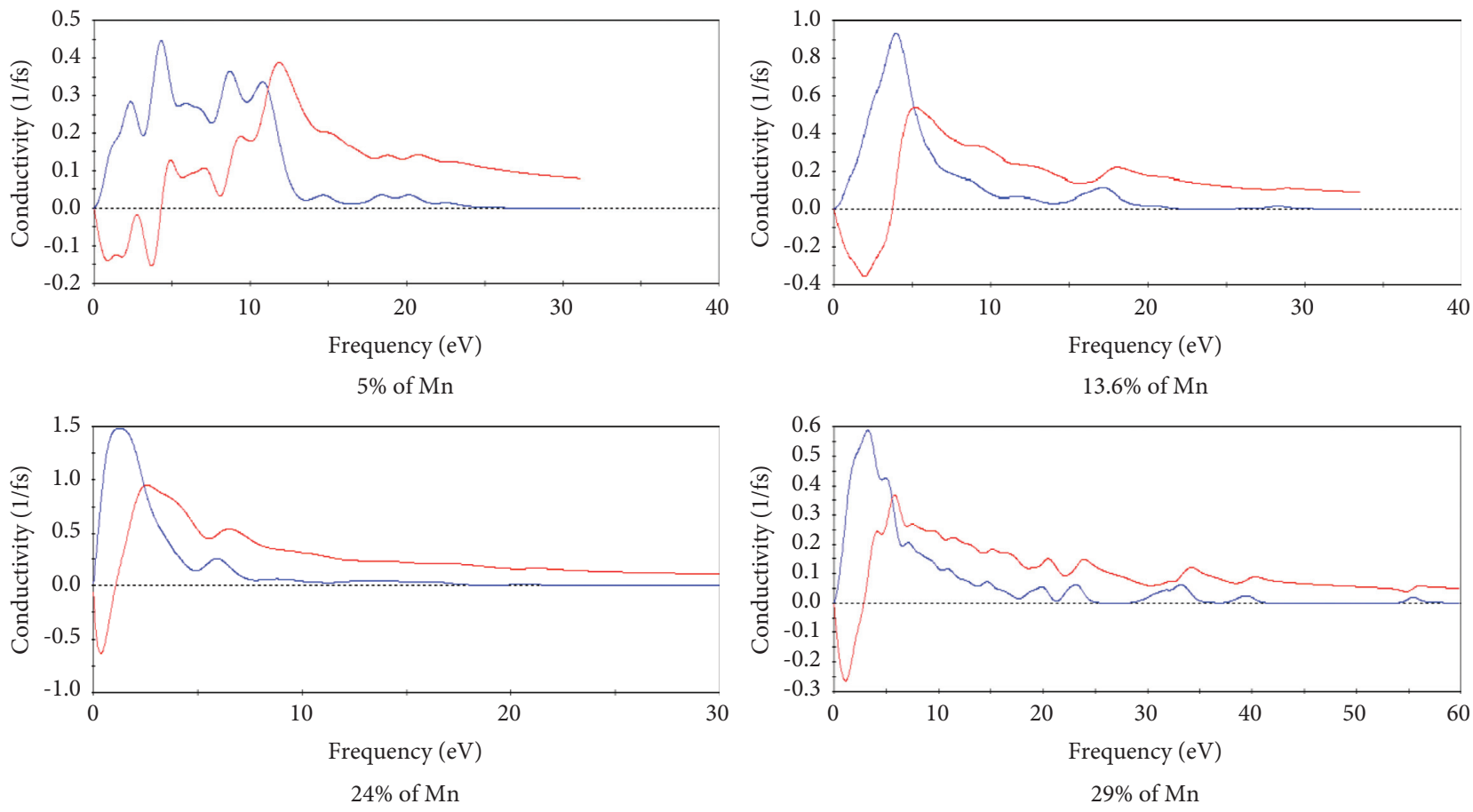

Figure 4: The conductivity of InSb-Mn with $\mathrm{Mn}=5 \%, \mathrm{Mn}=13.6 \%, \mathrm{Mn}=24 \%$, and $\mathrm{Mn}=29 \%$.

TABLE 1: The band gap of InSb-Mn with $\mathrm{Mn}=5 \%, \mathrm{Mn}=13.6 \%, \mathrm{Mn}=24 \%$, and $\mathrm{Mn}=29 \%$.

\begin{tabular}{lccr}
\hline Concentration of Mn & $5 \%(\mathrm{eV})$ & $13.6 \%(\mathrm{eV})$ & $24 \%(\mathrm{eV})$ \\
\hline Band gap & 0.407 & 0.341 & 0.033 \\
\hline
\end{tabular}

4.5. Density of States. Figure 1 represents the density of states of the prepared composite with varying doping concentrations. Density of states illustrates the occupancy of the electrons in the bands and sub bands. When the occupancy of the electrons in the band is high, then it allows more electrons to have the generation and recombination effect. Hence, the creation of more electron holes pair allows improved movement of the carriers in the structure. These effects of the occupancy of electrons in the bands and sub bands of the atom are due to the materials doping effects which change the atomic structure of the existing material. Hence, the effect of the doping will have significant improvement or decrement in the carrier concentration of the exiting material. In the present work, different doping concentrations of InSb-Mn are doped into the PMMA and its effective density of electrons. Depending on the varying doping concentration, the density of states graph structure varies with respect to the InSb-Mn doped PMMA. Peak intensity in the graph represented in Figure 1 illustrates the maximum electron occupancy. The peak intensity varies with respect to the varying doping concentration of the InSb-Mn. Doping of $24 \% \mathrm{Mn}$ has the much increase in the density of states than $5 \%$ of $\mathrm{Mn}$ and $13.6 \%$ of $\mathrm{Mn}$ concentrations, but further increase in the concentration of $\mathrm{Mn}$ to $29 \%$ results in decreasing density of states. In Figure 1, the maximum density of states at $24 \%$ of Mn concentration in thin film as illustrating the energy level of around $27.5 \mathrm{eV}$ with the peak intensity is shown.

InSb-Mn doped with PMMA with $24 \% \mathrm{Mn}$ has the much increase in the density of states than $5 \%$ of $\mathrm{Mn}$ and $13.6 \%$ of $\mathrm{Mn}$ concentrations but further increase in the concentration of $\mathrm{Mn}$ to $29 \%$ and then there is fall in the density of states. It is showing maximum density of states at $24 \%$ of Mn concentration in thin film.

\section{Conclusion}

Investigated first principle analysis of the electronic and optical properties of InSb-Mn doped with PMMA with varying $\mathrm{Mn}$ concentration reveals that the there is an enhanced electronic and optical property. The band gap decreases with increasing of Mn concentration. Similarly, the absorption of the InSb-Mn: PMMA shifts toward lower energy with the increase in Mn content. The optical conductivity is greater than those of pure InSb when $24 \%$ of $\mathrm{Mn}$ is doped but less than those of pure InSb doped with $29 \%$ of Mn. Thus, Mn doping plays an important role in the modulation of the optical absorption and optical conductivity. The band gap narrowing effect and the special optical properties suggest that the InSb-Mn: PMMA is a promising new semiconductor for optoelectronic applications. 


\section{Data Availability}

The data used to support the findings of this study are included in the article. Further data or information are available from the corresponding author upon request.

\section{Conflicts of Interest}

The authors declare that they have no conflicts of interest.

\section{References}

[1] M. Issai and M. Oshita, "Erratum: gabor lens focusing of a proton beam," Review of Scientific Instruments, vol. 61, p. $1556,1990$.

[2] L. P. Chen, J. J. Lou, T. H. Liu, Y. M. Pang, and S. J. Yang, "Evaluation of low dark current InSb photovoltaic detectors," Solid-State Electronics, vol. 35, no. 8, pp. 1081-1084, 1992.

[3] P. K. Chiang and M. Bedair, "p-njunction formation in InSb and InAs1-xSbxby metalorganic chemical vapor deposition," Applied Physics Letters, vol. 46, no. 4, pp. 383-385, 1985.

[4] R. K. Mangal, B. Triapthi, M. Singh, Y. K. Vijay, and A. Rais, "Growth and characterization of In-Sb thin film structure," Indian Journal of Pure \& Applied Physic s, vol. 45, p. 987, 2007.

[5] A. Okamoto, T. Yoshida, S. Muramatsu, and I. Shibasaki, "Magneto-resistance effect in InSb thin film grown using molecular beam epitaxy," Journal of Crystal Growth, vol. 201-202, pp. 765-768, 1999.

[6] B. R. Bennett, R. Magno, J. B. Boos, W. Kruppa, and M. G. Ancona, "Antimonide-based compound semiconductors for electronic devices:A review," Solid-State Electronics, vol. 49, no. 12, pp. 1875-1895, 2005.

[7] M. Razeghi, "Overview of antimonide based III-V semiconductor epitaxial layers and their applications at the center for quantum devices," The European Physical Journal - Applied Physics, vol. 23, no. 3, pp. 149-205, 2003.

[8] F. M. Li, G.-W. Hsieh, S. Dalal et al., "Zinc oxide nanostructures and high electron mobility nanocomposite thin film transistors," IEEE Transactions on Electron Devices, vol. 55, no. 11, pp. 3001-3011, Nov. 2008.

[9] Y. D. Park, J. A. Lim, Y. Jang et al., "Enhancement of the fieldeffect mobility of poly(3-hexylthiophene)/functionalized carbon nanotube hybrid transistors," Organic Electronics, vol. 9, no. 3, pp. 317-322, Jun. 2008.

[10] R. Farrow and D. Robertson: Patent Application No.WO1981GB0000057 Date of publication 1981-10-01.

[11] I. Kasai and J. R. Toman: Patent Application No US19940002502986 date of publication 1997-07-08.

[12] S. H. Lee: Patent Application No. US1997000918871 Date of publication 1999-01-19.

[13] T. Berus, M. Qszwaldowski, and J. Grabowski, "High quality Hall sensors made of heavily doped n-InSb epitaxial films," Sensors Axtuators A; Physical, vol. 116, no. 1, pp. 75-78, 2004.

[14] M. Szymonski, P. Korecki, P. Korecki, J. Kolodziej, P. Czuba, and P. Piatkowski, "Structure and electronic properties of ionic nano-layers MBE-grown on III-V semiconductors," Thin Solid Films, vol. 367, no. 1-2, pp. 134-141, 2000.

[15] M. M. Demir, M. Memesa, P. Castignolles, and G. Wegner, "PMMA/Zinc oxide nanocomposites prepared by in-situ bulk polymerization," Macromolecular Rapid Communications, vol. 27 , no. 10, pp. 763-770, 2006.

[16] T. Amine, "Investigation of the thermo-physical properties of poly(methyl methacrylate)-based Plexiglass to improve the performance of solar cells," Materials Science for Energy Technologies, vol. 4, pp. 349-356, 2021.

[17] A. A. Ahmad, A. M. Alsaad, Q. M. Al-Bataineh et al., "Synthesis and characterization of ZnO NPs-doped PMMABDK-MR polymer-coated thin films with UV curing for optical data storage applications," Polymer Bulletin, vol. 78, no. 3, pp. 1189-1211, 2021.

[18] T. H. AlAbdulaal and I. S. Yahia, "Optical linearity and nonlinearity, structural morphology of TiO2-doped PMMA/ FTO polymeric nanocomposite films: laser power attenuation," Optik, vol. 227, Article ID 166036, 2021.

[19] A. M. Alsaad, Q. M. Al-Bataineh, M. Telfah, A. A. Ahmad, Z. Albataineh, and A. Telfah, "Optical properties and photoisomerization processes of PMMA-BDK-MR nanocomposite thin films doped by silica nanoparticles," Polymer Bulletin, vol. 78, no. 7, pp. 3425-3441, 2021.

[20] D. Deepak and T. N. Ahipa, "Cyanopyridone doped PMMA films as UV and blue light filters: preparation and characterization," Optik, vol. 229, Article ID 166233, 2021.

[21] M. Wekwejt, S. Chen, B. Kaczmarek-Szczepańska et al., "Nanosilver-loaded PMMA bone cement doped with different bioactive glasses - evaluation of cytocompatibility, antibacterial activity, and mechanical properties," Biomaterials Science, vol. 9, no. 8, pp. 3112-3126, 2021.

[22] M. D. Segall, P. J. D. Lindan, M. J. Probert et al., "Firstprinciples simulation: ideas, illustrations and the CASTEP code," Journal of Physics: Condensed Matter, vol. 14, no. 11, pp. 2717-2744, 2002.

[23] M. C. Payne, M. P. Teter, D. C. Allan, T. A. Arias, and J. D. Joannopoulos, "Iterative minimization techniques forab initiototal-energy calculations: molecular dynamics and conjugate gradients," Reviews of Modern Physics, vol. 64, no. 4, pp. 1045-1097, 1992.

[24] http://www.tcm.phy.cam.ac.uk/castep/documentation/WebHelp/ content/modules/castep/thcasteppseudo.htm.

[25] J. P. Perdew, K. Burke, and M. Ernzerhof, "Generalized gradient approximation made simple," Physical Review Letters, vol. 77, no. 18, pp. 3865-3868, 1996.

[26] D. Vanderbilt, "Soft self-consistent pseudopotentials in a generalized eigenvalue formalism," Physical Review B, vol. 41, no. 11 , pp. 7892-7895, 1990.

[27] http://www.tcm.phy.cam.ac.uk/castep/documentation/WebHelp/ content/modules/castep/thcastepopticalprops.htm.

[28] http://www.tcm.phy.cam.ac.uk/castep/documentation/WebHelp/ content/modules/castep/dlgcastepdosoptions.htm.

[29] D. Nguyen-Manh, D. G. Pettifor, H. M. Sithole et al., "Electronic structure, pressure dependence and optical properties of FeS2," Materials Research Society Symposium Proceedings, vol. 491, p. 401, 1998.

[30] Y. Zeng and N. A. W. Holzwarth, "Density-functional calculation of the electronic structure and equilibrium geometry of iron pyrite (FeS2)," Physical Review B, vol. 50, no. 12, pp. 8214-8220, 1994.

[31] I. Opahle, K. Koepernik, and H. Eschrig, "Full-potential bandstructure calculation of iron pyrite," Physical Review B: Condensed Matter, vol. 60, no. 20, pp. 14035-14041. 